\title{
La diversification des stratégies résidentielles des élites à Varsovie après 1989
}

The diversification of the élites' housing strategies in Warsaw after 1989

Die Diversifizierung der Wohnraumstrategie der Eliten in Warschau nach 1989

\section{Caroline Bouloc}

\section{(2) OpenEdition}

\section{Journals}

Electronic version

URL: http://journals.openedition.org/rge/5904

ISSN: 2108-6478

\section{Publisher}

Association des géographes de l'Est

\section{Printed version}

Date of publication: 1 December 2016

ISSN: 0035-3213

Electronic reference

Caroline Bouloc, "La diversification des stratégies résidentielles des élites à Varsovie après 1989 ", Revue Géographique de l'Est [Online], vol. 56 / n³-4 | 2016, Online since 22 November 2016, connection on 08 September 2020. URL : http://journals.openedition.org/rge/5904

This text was automatically generated on 8 September 2020.

Tous droits réservés 


\title{
La diversification des stratégies résidentielles des élites à Varsovie après 1989
}

\author{
The diversification of the élites' housing strategies in Warsaw after 1989 \\ Die Diversifizierung der Wohnraumstrategie der Eliten in Warschau nach 1989
}

\section{Caroline Bouloc}

1 Après le rétablissement de la propriété privée et l'instauration d'un marché immobilier libéral, l'offre immobilière en Pologne s'est diversifiée proposant des types d'habitat différents destinés à des catégories de populations variées. Les classes supérieures ont été les premières à investir en dehors de l'habitat construit durant la période socialiste, de sorte que leurs concentrations spatiales sont moins fortes en 2002 qu'en 1988, provoquant un étalement urbain jusque dans les quartiers périphériques (Bouloc, 2011, 2015 ; Marczyńczak et al., 2012). A Varsovie, se renforcent d'une part les quartiers anciennement prestigieux et d'autre part de nouvelles zones au sein de la ville, éloignées du centre et encore peu peuplées. Qu'en est-il plus spécifiquement des élites, considérées comme les grandes gagnantes de la transformation? Existe-t-il un comportement résidentiel qui les distingue de la classe moyenne dans l'espace urbain?

2 Les recherches sur les catégories supérieures et plus spécifiquement sur les élites sont généralement portées par des sociologues analysant les changements sociaux du fait de la mondialisation ${ }^{1}$ et par des politistes travaillant sur leur lien avec le pouvoir ${ }^{2}$. Cependant, ces études analysent que trop rarement les trajectoires résidentielles et le rapport de ces classes au patrimoine résidentiel alors même qu'il est un des signes les plus fondamentaux de la distinction de classes (mis à part les travaux de M. Pinçon et M. Pinçon-Charlot, 1989, 1992, 1996, 2005a, 2005b). En géographie, les travaux portant sur les sphères supérieures sont rares et généralement anglophones (I. Hay, 2013)33. Quant aux travaux sur les élites polonaises, le constat est similaire, puisque qu'ils sont représentés dans le champ de la sociologie (entre autres : Słomczyński, 2002 et Zarycki, 2008), mais ne font l'objet que de quelques articles succincts en géographie sociale et urbaine (Liszewski, 1999 et Jałowiecki, 2000). 
3 Cet article réfléchit ainsi sur le rapport entre position sociale dominante d'un côté et patrimoine résidentiel et lieu d'habitation de l'autre. L'analyse des trajectoires résidentielles des élites varsoviennes permet non seulement de comprendre les changements sociaux qui sont à l'œuvre dans les espaces urbains post-socialistes, mais de proposer une définition de l'élite polonaise dépassant la différenciation classique selon les professions de celle-ci et en proposant une typologie originale se basant sur une analyse multicritères incluant les choix et préférences résidentielles. Ce travail de recherche repose ainsi sur des entretiens menés à Varsovie en 2011.

4 Dans une première partie, je précise la manière dont j'ai réalisé un échantillonnage représentatif en revenant sur la notion d'élite. Puis, afin de comprendre les choix résidentiels des élites varsoviennes d'aujourd'hui et d'identifier le type d'habitat qui les attire, j'exposerai la diversification de l'offre immobilière et des moyens d'accéder à la propriété qui ont accentué les inégalités socio-économiques dans l'espace après 1989. Ensuite, à la lumière des nouvelles dynamiques urbaines apparues, je présenterai les trajectoires et choix résidentiels des personnes interrogées ${ }^{4}$. Enfin, j'analyserai les divergences existantes au sein de ce groupe, proposant une typologie originale de l'élite varsovienne selon ces choix, préférences résidentielles et style de vie.

\section{De la notion d'élite à un échantillonnage représentatif}

5 L'utilisation du terme d'élite renvoie à une définition fonctionnaliste relativement ancienne se fondant sur la théorie de G. Mosca (1884) et de V. Pareto (1919), largement réinvestie à partir des années 1980. Par exemple, pour les sociologues $\mathrm{R}$. Boudon et $\mathrm{F}$. Bourricaud (1982), l'utilisation de ce terme permet d'expliquer la complexité de la société contemporaine. D’autres auteurs comme B. Jałowiecki (2000), J. Higley et J. Pakulski (2000) ou J. Higley et G. Lengyel (2000) transmettent une vision néolibérale de la société en prenant en compte strictement le rapport des élites au pouvoir. A cette vision s'oppose la théorie structuraliste de P. Bourdieu (1979) n'utilisant par le terme d' élite, mais celui de classe dominante. Ces individus se distingueraient du reste de la société par leurs statuts économique, social, culturel et symbolique. Cette définition est notamment réinvestie par T. Zarycki (2008) pour expliquer les changements structuraux au sein des élites d'Europe centrale. Afin de faciliter la lecture de ce travail, j'utilise le terme d'élite dans le sens de classe dominante définie par P. Bourdieu, alors que son emploi s'apparente généralement à une vision néolibérale de la société. J'assume cette confusion des genres, car j'essaie d'introduire dans le champ de la recherche française des principes développés dans la littérature polonaise (influencée en partie par la tradition anglophone). Ces héritages ne peuvent en effet être reniés, car la majorité des recherches qui s'interrogent sur les changements sociaux après 1989 se positionnent dans ce champ particulier. Il est ainsi nécessaire d'intégrer dans la réflexion ces deux démarches, bien qu'elles soient la plupart du temps antagonistes.

6 Le groupe étudié ne correspondant à aucune catégorie statistique définie, l'approche par analyse des données des recensements ne suffit guère. Approcher cette catégorie d'individus n'est pas non plus aisé (Pinçon et Pinçon-Charlot, 1989, 2005b). Il a donc été nécessaire d'utiliser la technique de proche en proche (Blanchet et Gotman, 2007), afin d'établir un échantillon suffisant permettant une analyse approfondie des trajectoires résidentielles de ces élites, de leurs choix et préférences résidentielles ainsi que de 
leurs pratiques de la ville. Au-delà d'une simple répartition spatiale des individus dans l'espace, il a été possible de comprendre les motivations sociales et économiques qui les poussent à préférer un lieu à un autre. Soixante-six entretiens semi-directifs ont ainsi été réalisés en 2011 avec des représentants de l'élite varsovienne ${ }^{5}$. Les variables qualitatives collectées regroupent des données socio-économiques, les lieux de résidences des personnes interrogées, leurs caractéristiques en termes de propriété, de préférences résidentielles et des pratiques spatiales. L'interprétation des résultats se fonde d'une part sur une analyse qualitative comme par exemple l'analyse des discours et d'autre part sur une analyse quantitative (comme les occurrences et l'analyse des correspondances factorielles).

7 Les individus interrogés sont pour la plupart des personnalités importantes connues à l'échelle nationale, reconnues au sein de leur milieu professionnel et côtoyant d'autres personnalités importantes et influentes. Ce milieu social est fortement enchevêtré. La technique de proche en proche l'a en effet fortement démontré, me permettant de rencontrer aussi bien des élites intellectuelles, artistiques et médiatiques que politiques ou encore économiques ${ }^{6}$. L'échantillon regroupait entre autres: des scénaristes et critiques de films et de séries télévisées, des membres du gouvernement haut placés, une présidente d'une fondation d'une multinationale, une directrice d'entreprise familiale de renommée nationale, des directeurs de filières de grandes multinationales et d'entreprises nationales, un courtier en bourse (trader) ou encore des exnomenklaturistes.

8 Ce groupe présente des caractéristiques socio-démographiques et économiques communes. Le statut d'élite reposant en grande partie sur la trajectoire professionnelle des individus, il est peu surprenant que notre échantillon ait pour plus de deux tiers plus de 41 ans. Ils appartiennent également aux catégories les plus riches de Pologne puisqu'en suivant les statistiques polonaises, la moyenne des salaires bruts par mois est de $3544 \mathrm{PLN}^{7}$, et celle de nos interviewés se situe entre 21801 PLN et 25000 PLN par mois, soit au minimum six fois la moyenne nationale. Si l'on prend la catégorie socioprofessionnelle ayant le salaire moyen brut le plus élevé, c'est-à-dire les directeurs et hauts cadres dans les secteurs privé et public, leurs salaires représentent encore au minimum trois fois le salaire moyen de cette catégorie. En outre, le revenu le plus bas se situe entre 3200 PLN et 4000 PLN, soit approximativement la moyenne nationale.

9 Par ailleurs, ils sont tous diplômés du supérieur : 70 \% ont acquis le diplôme de niveau Master ou celui d'Ingénieur, $29 \%$ sont docteurs. Outre un entre-soi marqué par le choix du conjoint exclusivement diplômé du supérieur (sauf pour deux cas), la continuité intergénérationnelle de classe est très forte puisque plus de trois quarts des individus interrogés ont des parents et un grand-père diplômés du supérieur. L'érudition est en effet un facteur significatif de distinction pour l'élite polonaise. Comme le démontre T. Zarycki (2008) dans ces travaux, l'élite a endossé un rôle d'opposition (politique) singulier dans l'histoire de la Pologne. Elle revêt ainsi un caractère symbolique et fondateur de l'identité nationale renforcée par des idéaux aristocratiques, de sorte que la transmission générationnelle a son importance. T. Zarycki replace en effet la conception de l'élite polonaise dans un contexte européen particulier. Bien que ces pays fussent centraux géographiquement, ils ne l'ont jamais été dans le système géopolitique et géoéconomique européen. De ce point de vue, ces régions périphériques se présentent avec un déficit en capital économique important qu'il a fallu combler par une autre sorte de capital, généralement par le capital culturel. L'élite polonaise se 
tourne alors « de préférence vers le capital culturel incorporé post-aristocratique (héritage du XIXe siècle), associé à des valeurs de la culture classique des pays occidentaux d'aujourd'hui $»^{8}$ (Zarycki, 2008, p. 63). Après plus de 25 ans de mutations politiques, économiques et sociales, cet héritage social et symbolique de l'élite polonaise pose question sur les trajectoires résidentielles de ces membres. Le patrimoine résidentiel étant en effet un des marqueurs les plus brutaux de la distinction de classes (Pinçon, Pinçon-Charlot, 2005b).

\section{Diversification de l'offre immobilière et des moyens d'accéder à la propriété}

10 Les changements politico-économiques du début des années 1990 ont eu un impact considérable sur la société polonaise et sur l'espace urbain. Les nouvelles dynamiques urbaines en œuvre dans les villes polonaises sont principalement issues de la réinstauration de la propriété privée, de la décentralisation et du rôle des collectivités locales dans l'aménagement du territoire, ainsi que de la tertiarisation de l'économie (WĘcławowicz, 1996, Enyedi, 1998). Les transformations les plus profondes concernent les centres-villes et les zones périphériques, réorganisant la structure générale de ces villes (Musil, 2005). Les centres-villes se sont en effet dépeuplés pour accueillir de nouvelles fonctionnalités principalement tertiaires et intégrer les sièges sociaux des entreprises (Śleszyński, 2012). Simultanément, les villes s’étendent en périphérie de manière exponentielle, de sorte qu'entre 1988 et 2002, $42 \%$ des citadins quittaient les grandes villes pour s'installer dans les communes limitrophes des villes centres (Lisowski, 2004). Les inégalités économiques se renforcent et la différenciation sociale par quartiers s'accentue, visible notamment par les différences de prix de l'immobilier. En effet, l'écart-type entre le prix moyen au mètre carré des logements neufs des différents quartiers de Varsovie a été multiplié par 2,3 entre 2005 et 2011.

11 Pour les élites, les changements dans le secteur de l'habitat vont avoir plusieurs conséquences dans leurs trajectoires résidentielles. C'est notamment à travers le processus de privatisation qu'elles vont acquérir un capital immobilier conséquent et creuser la différence avec les classes moyennes. La complexité de la structure du secteur résidentiel et des modalités d'occupation a multiplié les stratégies d'accession à la propriété pour les élites polonaises. La voie la plus rapide, sans complication juridique et statutaire, est sans nul doute l'accession à un logement nouvellement construit. A Varsovie, ces constructions ont réellement pris de l'essor durant les années 2000 et l'offre dans ce secteur de marché s'est extrêmement diversifiée (Coudroy de Lille, 2009, 2012; Górczyńska, 2012, Bouloc, 2013). Ces nouvelles constructions résidentielles se sont majoritairement concentrées dans les quartiers périphériques de la ville, là où les promoteurs immobiliers pouvaient débloquer de larges parcelles pour construire des résidences, mais aussi où les ménages pouvaient accéder à la maison individuelle. Ainsi, certains quartiers périphériques disposent d'un parc de logements pour plus de la moitié construit entre 2002 et 2012 (Bouloc, Górczyńska, 2014).

12 Par ailleurs, de nombreux Polonais sont devenus propriétaires grâce à la politique de privatisation du parc de logements communal. Les collectivités locales, auxquelles incombe la responsabilité de ces logements depuis 1990, ont vendu une grande part du stock à ses locataires à environ $10 \%$ du prix du marché permettant aux ménages aux revenus modestes d'accéder à la propriété. Pour de nombreuses élites plus âgées, cette 
politique a été une première étape d'accession à la propriété dès le milieu des années 1990.

Enfin, la restitution de logements anciens à leurs propriétaires d'origine est également un moyen pour certaines élites ayant des origines sociales urbaines de récupérer des biens immobiliers au cœur de la ville. Ces restitutions, quasi-inexistantes durant les années 1990, ont pris de l'ampleur depuis les années 2000. A Varsovie, ce phénomène a été dans un premier temps plus timide qu'à Cracovie, mais les demandes de restitutions ne cessent d'augmenter (Bouloc, Górczyńska, 2014). Il n'existe actuellement aucune loi facilitant la restitution de ces biens immobiliers de sorte que les procédures s'éternisent très souvent sur plusieurs années (Glock, 2007). Par ailleurs, ces restitutions ont conduit à la superposition des différents statuts d'occupation rendant également la gestion de l'immeuble difficile (Górczyńska, 2013).

La diversification de l'offre immobilière et la multiplication des moyens de devenir propriétaire ont permis de rendre plus mobiles les classes les plus aisées. Après plus de 25 ans de transition sociale et urbaine, de nouvelles logiques résidentielles se dessinent-elles au sein de l'élite varsovienne?

\section{Quelle localisation et quel habitat pour l'élite varsovienne?}

\section{A. Les trajets résidentiels de la réussite}

Les trajets résidentiels des élites varsoviennes interrogées durant la période socialiste se ressemblent à peu près toutes. Cette période, caractérisée par une pénurie constante de logements (la demande augmentant plus vite que la réserve du nouveau stock), l'attente d'attribution était extrêmement longue (Regulska, 1987 ; Ciechocinska, 1987 ; Coudroy de Lille, 2006). Or, pour une grande partie des personnes interrogées, ce délai dépassait rarement cinq ans, contrairement à certains qui attendaient plus de dix ans. Le changement d'appartement était également un fait rare, pourtant les trajectoires résidentielles des élites interrogées démontrent le contraire, puisqu'ils ont déménagé plus fréquemment que la majorité des Polonais grâce à une promotion professionnelle ou à leurs connaissances au sein du Parti. Malgré tout, ils ont tous vécu dans de grands ensembles (excepté neuf d'entre eux) qui venaient d'être construits au moment de leur emménagement.

16 Après 1989, la mobilité résidentielle a été plus conséquente. Vers 1993, pratiquement tous ceux qui logeaient dans un grand ensemble avaient déménagé dans un logement neuf. Seuls les plus âgés, dont les immeubles étaient situés dans de bons quartiers, sont restés. Ceux qui résidaient dans un bâtiment d'avant-guerre y sont également majoritairement restés. Ces déménagements se sont pour une grande majorité dirigés vers un logement en maison individuelle neuve. Tous ont également changé de logement pour gagner en surface, en confort, voire en luxe.

\section{B. Les lieux de résidences actuels}

17 De manière générale et contre toute attente, la ségrégation sociale dans les villes polonaises s'est dans un premier temps atténuée, du fait de la forte mobilité résidentielle des catégories supérieures durant les années 1990 (Bouloc, 2011, 2013 ; 
Marczyńczak et al., 2012, 2014). A Varsovie, les données du recensement de 2002 présentaient une concentration des classes supérieures le long d'un corridor nord-sud sur la rive gauche de la Vistule. Cette concentration s'est prolongée vers le nord à Bielany et vers le sud à Ursynów et Wilanów. Par ailleurs, elle s'est disséminée vers les quartiers périphériques comme par exemple à Wawer (Bouloc, 2013). A première vue, les élites interrogées suivent les mêmes dynamiques (Fig. 1). Le corridor nord-sud est bien visible, avec une plus forte concentration en centre-ville et une migration vers le sud-est, du côté de la rive droite de la Vistule de Saska KĘpa à Aleksandrów.

Figure 1 : Lieux de résidence des personnes enquêtées selon leurs types (carte et schéma)

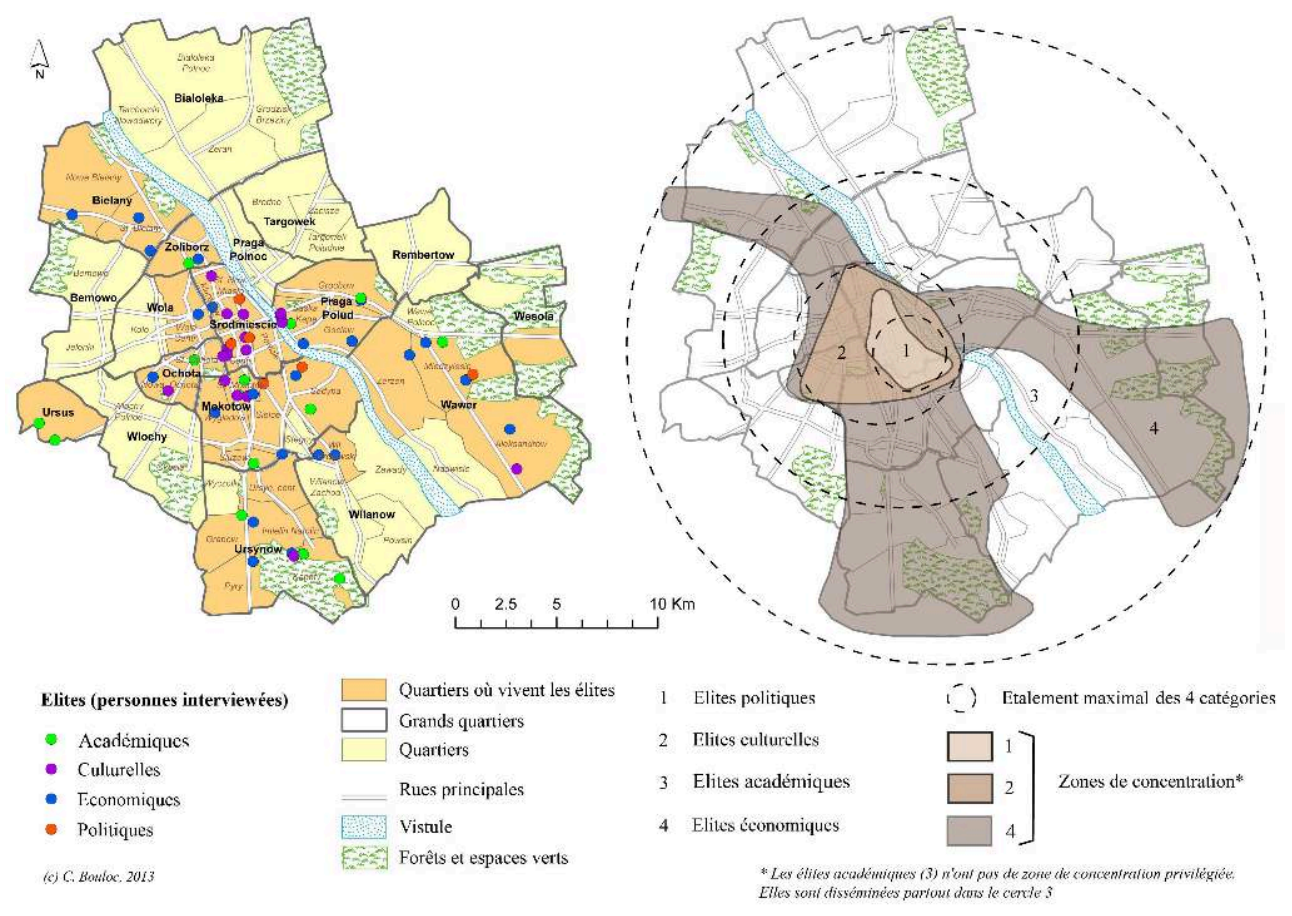

Source: Entretiens réalisés en 2011

Au-delà de ces logiques extrêmement générales, des dynamiques propres à chaque fraction de l'élite se dessinent. En effet, les élites politiques en règle générale résident dans le centre resserré (1), non loin des ministères et places de pouvoir. Ensuite, les élites culturelles (artistiques) se situent dans le centre-ville, dépassant rarement ses limites, ou seulement dans le vieux Mokotów, le vieux Żoliborz et le Saska K巨̨pa pavillonnaire (2). Les élites académiques (3) ont un comportement résidentiel aux logiques très disparates d'un interviewé à l'autre, à cause de leur manque de capital économique, de sorte que tous usent de stratégies afin de ne pas trop s'éloigner des zones de résidence de prestige. Enfin, les élites exclusivement économiques ont une large préférence pour la périphérie (4) : à partir des quartiers péricentraux comme Ursynów, Mokotów ou encore Saska KĘpa, jusqu'à des quartiers en cours de construction tels que Anin (Wawer) et Wesola, Stare Bielany et Ursynów. Cette typologie présente cependant certaines limites, car d'autres processus et dynamiques sont perceptibles au sein de ce groupe étudié. 


\section{Vivre en banlieue ou au cœur de la ville ?}

19 A partir des années 1990, une partie des Varsoviens souhaitant accéder à la propriété et à la maison individuelle ont décidé de s'installer dans la banlieue plus ou moins proche de Varsovie. Plus de trois quarts des communes composant l'espace métropolitain de Varsovie ont vu leur surface résidentielle augmenter de plus de $200 \%$ entre 2000 et 2004 (Degórska, Derణgowska, 2008). Ces dynamiques se sont encore accentuées entre 2004 et 2010 (Degórska, 2012) et concernent principalement les classes moyennes et supérieures, ayant les moyens d'accéder à la propriété ainsi que de posséder une ou deux automobiles pour effectuer les allers-retours journaliers entre la ville-centre et leur domicile (Komornicki, 2011).

Un sixième des personnes interrogées déclarent vouloir vivre en banlieue qu'elles considèrent comme un lieu idéal (« loin du bruit de la ville»). La majorité ne se décide pourtant pas à y emménager. Ensuite, un peu plus d'un quart déclarent avoir une préférence de vie pour les quartiers périphériques de Varsovie qui ont la particularité d'être très peu urbanisés et encore forestiers. Ce sont des espaces qui ont l'avantage de présenter tous les atouts de la banlieue (maisons individuelles, vastes jardins) en restant dans les limites administratives de la ville. Ce sont les quartiers péri-centraux qui remportent cependant tous les suffrages pour leur proximité au centre-ville. De manière plus précise, l'accès à la maison individuelle s'apparente au style de vie idéal, puisque plus de deux tiers d'entre eux la préfèrent à un appartement leur rappelant les logements de l'époque socialiste.

21 Le choix de vivre en banlieue ou en ville est également une question de génération, car au-delà des effets de classe sociale, les aspirations dues à l'âge de l'individu ainsi que sa situation matrimoniale sont primordiaux dans le choix et la préférence de localisation du centre-ville à la banlieue. Ainsi, les $20-35$ ans ont une préférence pour le centreville pour des questions principalement de loisirs. Les $35-60$ ans ont soit une préférence pour la banlieue, soit une affection pour les quartiers péricentraux. C'est dans cette tranche d'âge que la réalisation des aspirations de classes est visible, alors que les individus s'installent durablement dans des trajectoires de vie. Au-delà de 60 ans, les logiques résidentielles privilégient la ville-centre.

\section{Etre propriétaire, une manière de se distinguer}

\section{Etre propriétaire d'un ou de plusieurs logements}

Après 1989, les aspirations des individus à la propriété individuelle ont été extrêmement importantes, de sorte que le pourcentage des logements privés dans le secteur de l'habitat n'a cessé d'augmenter passant de $37 \%$ en 2002 à $52 \%$ en 2011 dans les villes polonaises (Bouloc, Górczyńska, 2014). Si l'on ajoute les coopératives en propriété privée ( $22 \%$ en 2011), le secteur privé de l'habitat est devenu la norme pour un grand nombre de Polonais aujourd'hui. Il n'est donc pas étonnant que la totalité de nos interviewés soient propriétaires de leur logement de résidence. Ce n'est pas tant le fait de posséder le logement dans lequel ils résident qui les fait se distinguer, mais plutôt le nombre de propriétés dont ils disposent. Deux tiers d'entre eux sont propriétaires d'au moins deux logements. Ce sont principalement les élites économiques qui disposent de plusieurs logements et les élites académiques qui en 
disposent le moins. La génération qui est la mieux dotée aujourd'hui en investissement immobilier est celle se situant entre 51 et 60 ans.

\section{L'élite se distingue-t-elle par le type de bâtiment qu'elle choisit ?} première, et surtout celle qui a prévalu durant les années 1990, est l'acquisition d'un logement neuf; phénomène bien évidemment lié à la modernisation et à l'offre immobilière plus diversifiée et de meilleure qualité qui s'est présentée à partir du milieu des années 1990. La seconde, plus récente et se développant durant la deuxième moitié des années 2000, s'apparente à la recherche de logements anciens datant d'avant-guerre, du fait de leur rareté et donc de leur exception. Ce nouvel élan est quelque peu tardif à cause de la complexité dans certains cas d'acquérir un logement ancien, mais surtout du fait que $47 \%^{9}$ des logements d'avant-guerre sont encore communaux, et ce milieu social ne convient généralement pas aux élites interrogées. L'attrait pour les anciens bâtiments est donc timide et concerne exclusivement les anciens quartiers prestigieux : Śródmieście, Żoliborz, Mokotów et Saska KĘpa.

\section{E. La résidence fermée : un phénomène d'habitat élitaire ?}

Au même titre que dans les métropoles d'Europe de l'Ouest (Le Goix, 2002, 2006 ; Billard et al., 2011 ; Madoré, 2009), les résidences fermées ont fait leur apparition dans les villes d'Europe centrale (GĄsior-Niemiec et al., 2007 ; Czéfalvay, 2011 ; Górczynska, 2012). Ces nouvelles configurations du bâti urbain sont principalement faites pour accueillir les catégories supérieures, comme l'indiquent Jałowiecki et al. (2004) dans leurs enquêtes sur ce type d'appartements. En effet, ce sont principalement les directeurs et les managers qui peuplent les résidences fermées du centre-ville ${ }^{10}$. Dans cette mesure, les élites, en tant que haut de la classe supérieure, devraient principalement choisir ce nouveau type de résidences sécurisées. Que révèlent les entretiens? Contre toute attente, seulement un tiers des personnes interrogées vivent dans une résidence fermée et un peu plus d'un quart déclarent aimer ces mêmes résidences. La nouvelle résidence fermée luxueuse n'est donc pas le type d'habitat recherché en priorité par les élites, elle peut être une étape pour certains, mais c'est la maison individuelle, isolée de tous à laquelle ils aspirent le plus fortement.

Les élites interrogées ont été extrêmement mobiles après 1989 cherchant à "s'enfuir » de l'habitat socialiste, mais restant attachées aux quartiers anciennement prestigieux.

Revue Géographique de l'Est, vol. 56 / n³-4 | 2016 
L'habitat neuf est fortement privilégié, même si un intérêt grandissant envers l'habitat d'avant-guerre est perceptible. Pourtant, au-delà de ces logiques communes, des distinctions de comportements et de choix résidentiels s'exercent au sein de cette catégorie présentant des habitus différents d'un groupe à l'autre.

\section{Typologie des élites selon leurs comportements socio-spatiaux et leurs choix résidentiels}

La typologie classique selon le type de professions pour les élites explique certaines localisations, mais est bien trop simpliste pour véritablement définir la complexité des comportements socio-spatiaux et les choix résidentiels dans leur globalité. Une étude plus approfondie est nécessaire notamment par la prise en compte des choix, des préférences et des aspirations résidentielles. A cet égard, une analyse factorielle des correspondances (AFC) semble être la méthode la plus appropriée afin de mettre en relief les différents aspects révélés par les entretiens. Cette méthode résume l'information sur des axes factoriels croisés permettant une interprétation des données ${ }^{11}$ (Dumolard, 2011).

Le nuage de points ainsi réalisé présente un pourcentage de confiance de près de $15 \%^{12}$, et révèle une typologie des élites assez singulière dont la différenciation entre les individus se fait majoritairement selon la richesse (axe vertical) et selon l'âge des individus et du bâti (axe horizontal) (Fig. 2). Cinq groupes ont ainsi pu être définis et chacun a des spécificités qui lui sont propres et dont les choix symboliques sont ancrés dans une logique de classe ou de sous-classe.

Figure 2 : Typologie des élites selon des caractéristiques socio-économiques, démographiques, d'habitation, de choix de vie et de préférences (AFC)

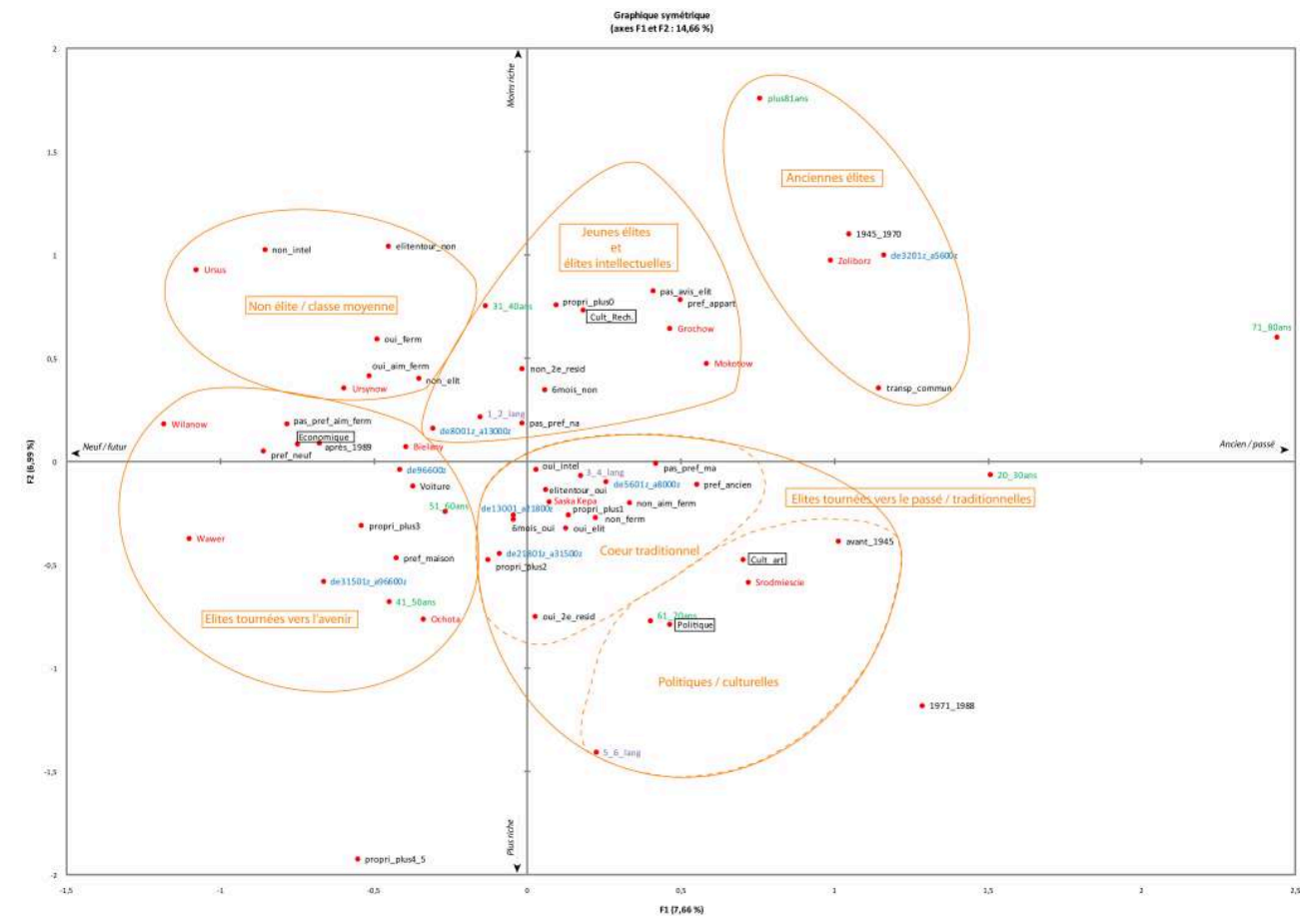

Source: Entretiens réalisés en 2011 


\section{A. Les anciennes élites : une sauvegarde du statut par l'habitat socialiste}

29 L'ancienne élite rassemble les individus qui ont été des élites durant la période socialiste grâce à leur statut socio-professionnel, mais qui étant trop âgés n'ont pas pu convertir leurs capitaux culturel, politique et social après 1989 en capital économique. Leur retraite est supérieure à celle de la moyenne polonaise des retraites, mais cela ne leur permet pas de quitter les logements construits entre 1945 et 1970.

Figure 3 : Immeubles construits durant la période socialiste à Żoliborz
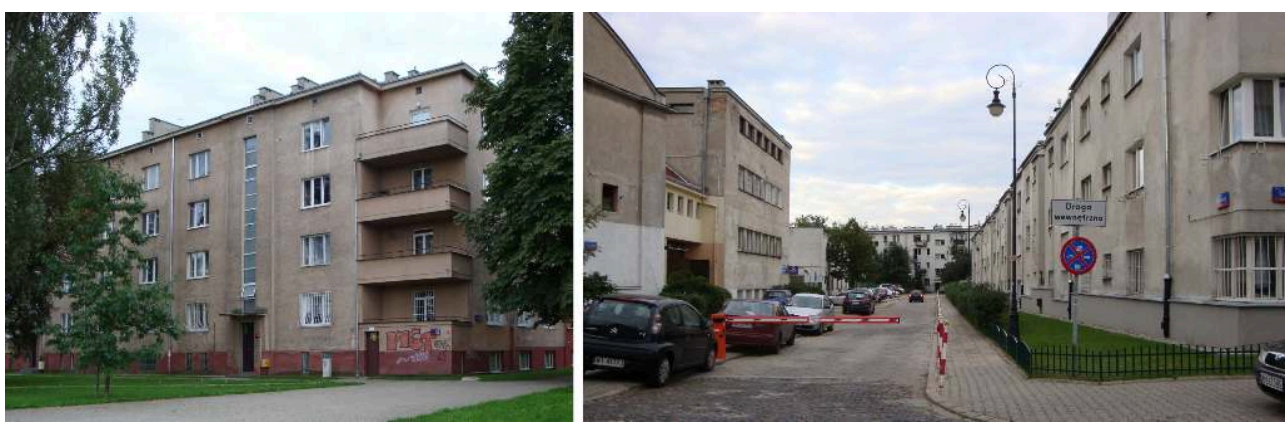

Auteur: C. Bouloc

Le lieu d'habitation est pour ce groupe très important, car ces membres résident tous en majorité dans les quartiers centraux ou péri-centraux. Żoliborz est un des quartiers le plus représentatif regroupant ce type d'élites (Fig. 3), car les coopératives $\mathrm{d}^{\prime}$ habitation ${ }^{13}$ ont énormément construit dans ce quartier dans les vingt premières années de l'époque socialiste. Cette place de choix leur permet d'avoir accès à toutes les infrastructures (surtout publiques) et, ne disposant pas de voiture, leurs déplacements sont facilités par les transports en commun très développés dans ces anciens quartiers. Ces quartiers ont également l'avantage d'accueillir des élites plus récentes ayant assez de capital économique pour rénover d'anciens bâtiments d'avant-guerre (Fig. 4). Les quartiers périphériques et la banlieue couperaient les anciennes élites de leurs vies sociales (ou mondaines), notamment à cause du coût du déplacement, car ces liens restent fragiles notamment parce qu'ils ne sont plus actifs professionnellement.

Figure 4 : Maisons individuelles d'avant-guerre rénovées à Żoliborz
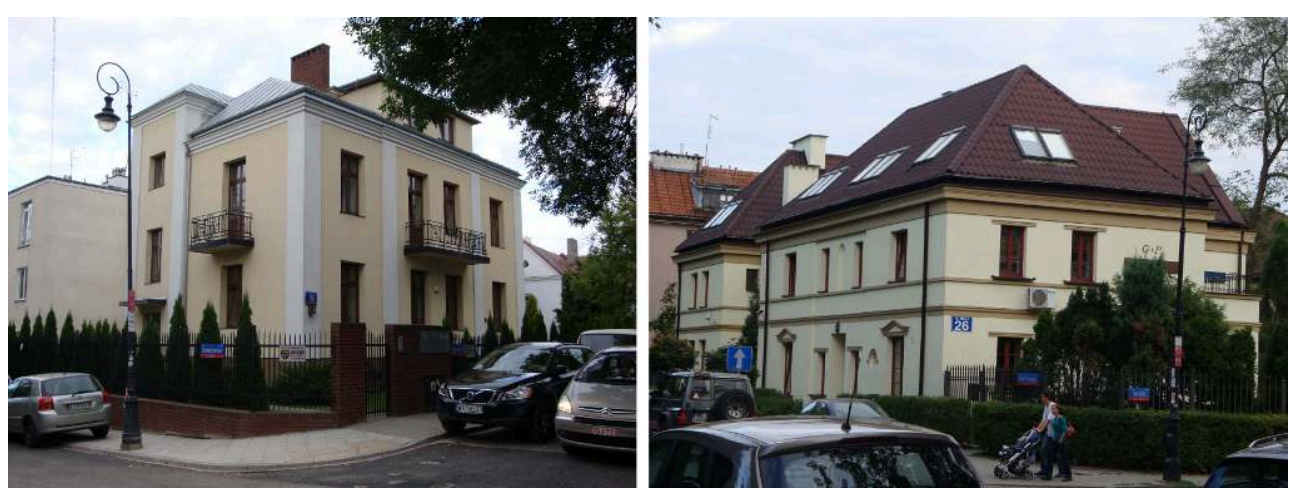

Auteur: C. Bouloc 


\section{B. Les jeunes élites et les élites intellectuelles : un manque de capital économique}

31 Les jeunes élites (entre 20 et 40 ans) ne sont pas très éloignées dans leur comportement des anciennes élites, car leurs stratégies de regroupement ou d'aspiration de classe doivent contourner la même difficulté : le manque de capital économique. Leurs salaires, quoique plus élevés que la moyenne nationale, sont inférieurs au salaire moyen de nos interviewés. Ces différentes caractéristiques les positionnent à michemin entre les autres fractions de l'élite. Si les choix et les préférences sont déjà perceptibles dans leurs discours, ils ne sont pas encore suivis d'actions concrètes (comme l'achat de logements). Seul le quartier de Mokotów reste fortement ancré dans les choix résidentiels. En effet, ce quartier péricentral semble attirer très fortement les plus jeunes qui le définissent souvent comme très dynamique, car il est le prolongement direct du centre-ville actif et en «ébullition ${ }^{14}$.

Cette catégorie regroupe également les élites académiques dont le revenu les empêche d'accéder à des logements luxueux. Ces derniers ont principalement privilégié les quartiers anciennement prestigieux, vivant dans des bâtiments construits durant le socialisme, plutôt que des logements excentrés en périphérie dans des quartiers de mauvaise réputation. C'est pourquoi ils ont souvent du mal à se considérer en tant qu'élite, car leur niveau de vie s'apparente plus à la classe moyenne, mais leurs aspirations professionnelles et culturelles sont plus élevées, ambitionnant d'intégrer la classe dominante et donc choisissant des lieux d'habitation centraux. Le capital symbolique surpasse encore le capital économique dans la définition de l'élite polonaise (voir première partie) et c'est à cet égard qu'ils peuvent être qualifiés d'élite à part entière.

\section{Les élites traditionnelles : symboliques de l'élite dans son ensemble}

33 Un autre groupe d'élites se distingue prioritairement par son capital symbolique, ce que l'on pourrait donc qualifier d'élite traditionnelle faisant référence à des positions sociales et des habitus issus de l'époque d'avant-guerre. Dans cette catégorie, il est possible d'identifier un cœur traditionnel, très proche de l'origine (donc du profil moyen ${ }^{15}$ ) dont toutes les élites ont tendance à s'inspirer et qui est au centre de la définition de leur statut. Les individus de cette catégorie se définissent volontiers comme membres de l'intelligentsia polonaise et utilisent plus rarement le terme d'élite pour se définir. Leurs préférences d'habitation sont principalement tournées vers cette symbolique passée, préférant l'habitat ancien et n'aimant pas les nouvelles constructions. Un accent particulier est porté sur l'histoire résidentielle familiale avant la Seconde Guerre mondiale.

Le quartier représentant le mieux ces différentes caractéristiques liées à la continuité des traditions de l'intelligentsia et de l'aristocratie polonaise est Saska KĘpa, dont les maisons ont en grande partie survécu après la guerre et se situant presque en centreville; il attire un grand nombre d'élites à l'heure actuelle (Fig. 5). Par ailleurs, les élites dites politiques s'éloignent du profil commun notamment par leur fonction qui les 
oblige (pour les plus hauts placés d'entre eux) à vivre en centre-ville (Śródmieście), tout comme les élites culturelles.

Figure 5 : Habitats caractéristiques à Saska Kępa
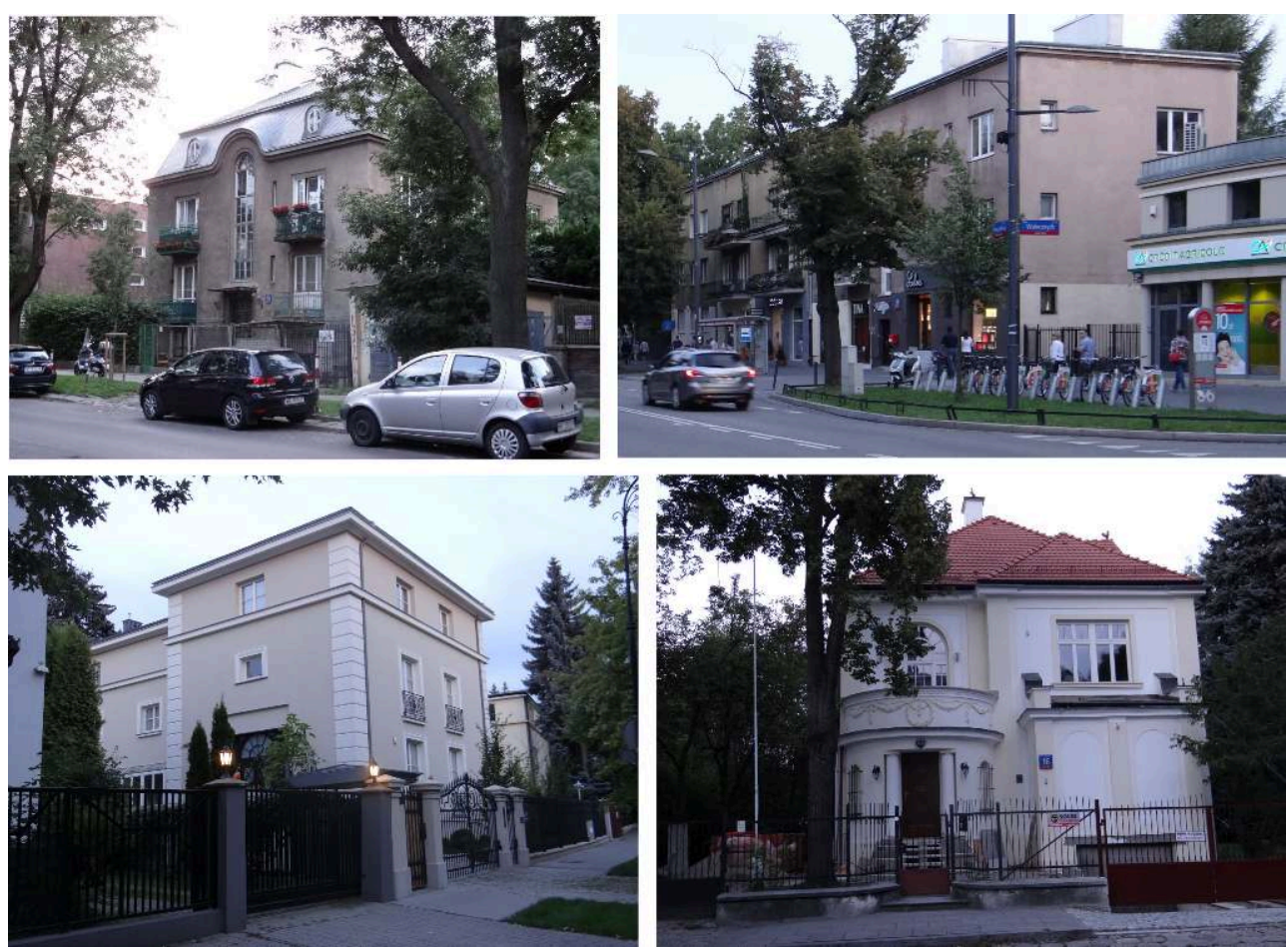

Auteur: C. Bouloc

\section{Les élites tournées vers l'avenir : une préférence pour l'habitat neuf}

Les élites tournées vers l'avenir regroupent les individus qui ont une préférence assez marquée pour tout ce qui a été initié après 1989. En effet, ils ont une inclinaison pour l'habitat neuf et pour la maison individuelle. Il s'agit des plus riches des individus interrogés (au sommet des revenus, bien supérieurs à la moyenne). Ces individus sont en plein épanouissement de leurs activités professionnelles. Agés entre 41 et 60 ans, ils disposent d'au moins trois propriétés en plus de celle dans laquelle ils résident. Ils vivent principalement dans les quartiers périphériques de la ville comme Wawer, Wilanów, ou péricentraux comme Ochota (Fig. 6). En revanche, ces élites n'ont pas particulièrement d'avis sur les résidences fermées, car elles n'ont pas été une étape de leur vie, ni un besoin particulier actuel, leur richesse rendant possible la surveillance de leur maison individuelle. 
Figure 6 : Exemple de maisons individuelles à Wawer (Anin)
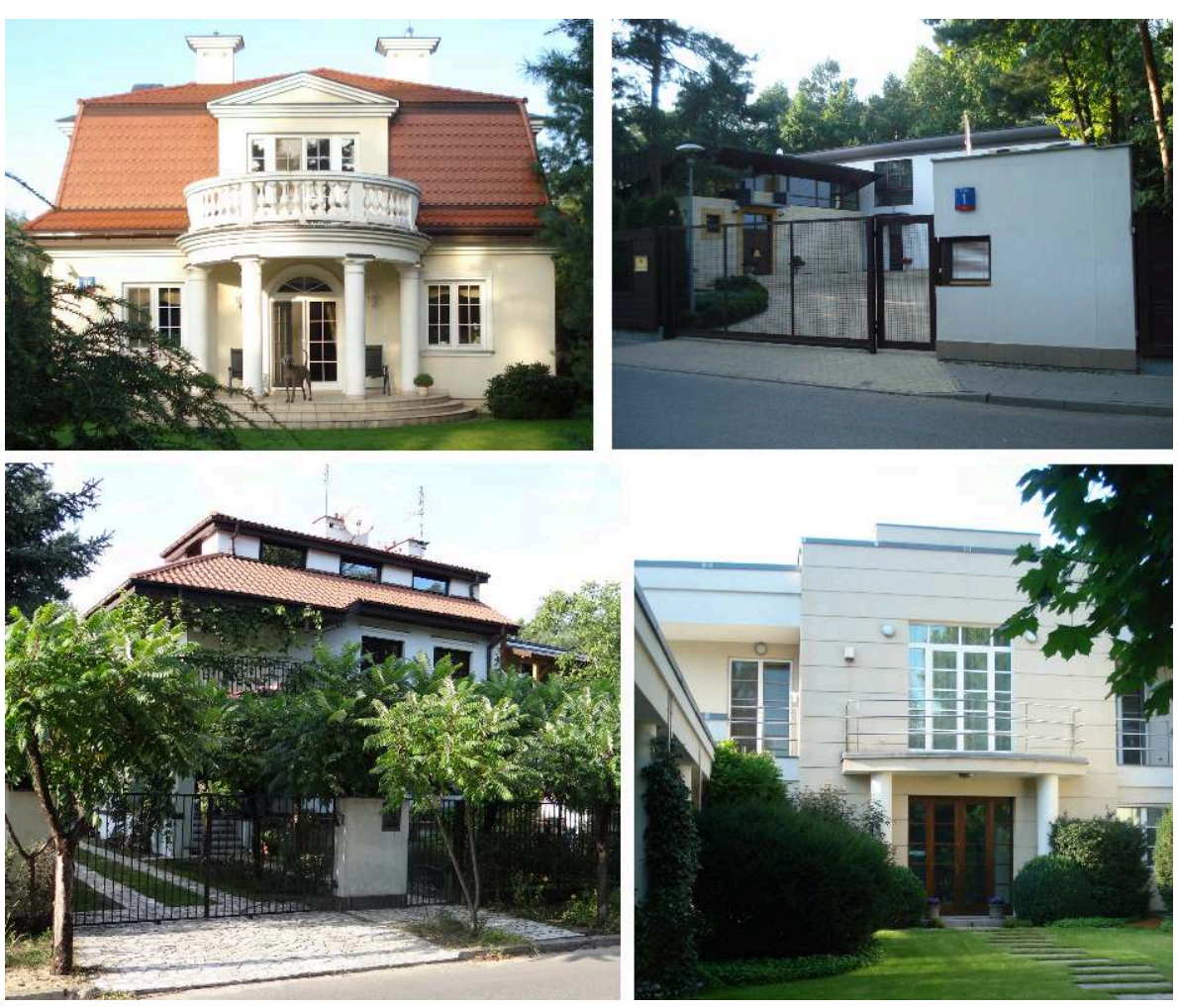

Auteur : C. Bouloc

36 Les profils de ces élites se ressemblent beaucoup; elles disposent toutes d'une forte influence dans leurs milieux professionnels (au plus haut de la hiérarchie), sont extrêmement actives et désirent plus que tout une résidence principale éloignée du «brouhaha du centre-ville» où elles passent le plus clair de leur temps. Leurs habitus s'apparentent de plus en plus à ce que B. Jałowiecki (2004) appelle la classe métropolitaine, notamment par leur mode de vie et leurs ambitions professionnelles. Cependant, ces élites n'en restent pas moins influencées par le cour traditionnel notamment de par leur éducation et leur ascendance familiale se déclarant bien volontiers issus de l'intelligentsia ou de lignées aristocratiques. Ils semblent vouloir malgré tout s'en dégager notamment parce qu'ils se définissent comme des élites au niveau national, mais plus rarement et plus difficilement comme membre de l'intelligentsia aujourd'hui.

\section{E. La classe moyenne supérieure : une non-élite?}

Etant donné que le choix des individus interrogé s'est effectué par l'intermédiaire de critères les plus larges possibles (voir première partie) et suivant la technique de proche en proche, certains ont un habitus qui s'apparente plus à celui de la classe moyenne supérieure du fait de leurs comportements sociaux, des préférences et des choix d'habitation. Ce sont eux qui principalement préfèrent et vivent dans les résidences fermées, donc sont attirés par les logements neufs qui permettent de les différencier de la classe moyenne (Fig. 7). Deux quartiers apparaissent comme des lieux caractéristiques de cette catégorie d'individus: Ursynów et Ursus. Le second est beaucoup plus équivoque que le premier, car en effet Ursynów se situe à la limite du 
groupe classe moyenne supérieure, proche de celui des élites tournées vers l'avenir, car une partie du quartier (Kabaty et Pyry) est pavillonnaire et attire ainsi ces élites. Ursus quant à lui, par son passé ouvrier, tend à attirer plus particulièrement dans sa partie pavillonnaire la classe moyenne ascendante.

Figure 7 : Résidence fermée Marina à Mokotów
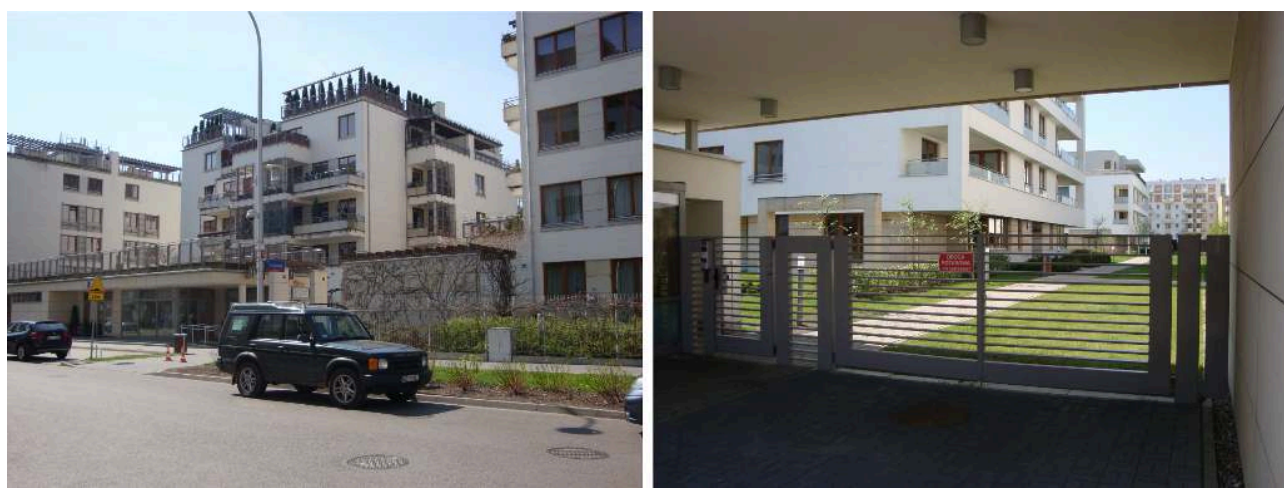

Auteur: C. Bouloc des principaux moyens de se distinguer socio-économiquement pour les élites varsoviennes. Le choix des lieux de vie dans la ville, du quartier d'habitation, n'est donc pas anodin et bien qu'il s'agisse d'une démarche individuelle, elle est régie (consciemment ou inconsciemment) par des aspirations de classe. A la lumière des transformations urbaines, les élites varsoviennes de par leurs activités professionnelles ne vivent pas au-delà des limites administratives de la ville, disposent de plusieurs propriétés et ne sont pas enclines à vivre dans une résidence fermée. Malgré tout, des stratégies distinctes se sont élaborées selon les catégories d'élites auxquelles l'individu interrogé appartient, car en effet suivant la structure des différents capitaux qui font leur individualité sociale, les choix et les stratégies de regroupement ou d'isolation seront différents. 
41 La typologie présentée ici a permis de croiser les habitus sociaux de classes liés aux différents types d'élites avec les comportements résidentiels de ceux-ci. Ce faisant, par l'exemple polonais, la définition de l'élite nous semble bien plus complexe et plus hétérogène qu'une simple délimitation en termes de statut professionnel. Elle dépend au contraire des comportements sociaux et des choix de vie (du «goût » des élites pour certaines choses plus que pour d'autres). Ces mécanismes permettent de prendre en compte l'imbrication des différents capitaux entre eux comme l'importance dans cet exemple que peuvent revêtir les capitaux symbolique et culturel pour les élites traditionnelles, et à l'inverse le rôle que peut jouer la maison individuelle comme symbole fort de renouveau et de réussite pour certaines élites dans la société polonaise d'aujourd'hui. L'habitat élitaire polonais n'est donc pas unilatéral et seulement synonyme de richesse économique, d'autres processus de distinction sont à l'œuvre. La résurgence du capital économique après 1989 a permis aux capitaux culturel et symbolique de prendre toute leur envergure dans le choix de l'habitat des élites.

\section{BIBLIOGRAPHY}

Billard G., Chevalier J., Madoré F., Waillat F., 2011, Quartiers sécurisés. Un nouveau défi pour la ville?, Editions Les Carnets de l'info, collection « Modes de ville».

Blanchet A., Gotman A., 2007 (réed.), L'enquête et ses méthodes : L'entretien, Armand Colin, Paris. Boudon R., Bourricaud F., 1982, Dictionnaire critique de la sociologie, P.U.F., Paris, (définition des élites).

Bouloc C., 2011, « Urban Elites in Polish intra-metropolitan space: the case of Warsaw, Krakow and Lodz", in: Kalogirou S. (eds.), ecQTG 2011 - 17th European Colloquium on Quantitative and Theorical Geography-Proceedings, 2-5 September 2011, Greek Society for Demographic Studies, Harokopio University, Geography department, No.33, p. 42 -51.

Bouloc C., 2013, Les élites dans les villes polonaises. Etude de géographie sociale, thèse co-dirigée par P. Petsimeris et G. WĘcławowicz, Université Paris 1, Académie des Sciences de Pologne, soutenue le 7 décembre 2013 à Paris.

Bouloc C., Górczyńska M., 2014, «L'accession à la propriété privée dans les villes polonaises : un défi de transition post-socialiste », EchoGéo [En ligne], 30 | 2014, mis en ligne le 31 décembre 2014, URL : http://echogeo.revues.org/14057 ; DOI : 10.4000/echogeo.14057

Bourdieu P., 1979, La distinction, critique sociale du jugement, Les éditions de minuit, collection Le sens commun, Paris.

Ciechocinska M., 1987, “Government interventions to balance housing supply and urban population growth: the case of Warsaw", International Journal of Urban and Regional Research, 11 (1), p. $9-26$.

Coudroy de Lille L., 2006, "La lutte des places: les élites et leurs territoires dans les villes de la Pologne communiste", in N. Bauquet, F. Bocholier (dir.), Le communisme et les élites en Europe centrale, Les rencontres de Normale Sup', éd. Ens rue d'Ulm, Puf, Paris, p. 253-270. 
Coudroy de Lille L., 2009, La construction de logements à Varsovie depuis 1990 : une reprise en demi-teinte, in Berard E., Jaquant C. (dir.), Berlin-Varsovie-Moscou, L'architecture au-delà du mur, Picard, Paris, p. 203-220.

Coudroy de Lille L., 2012, L'habitat du « post » en Europe centrale et orientale, Historiens et géographes, $n^{\circ}$ 419, Editeur APHG, Paris, dossier p. 75-80.

Czéfalvay Z., 2011, "Gated Communities for Security of Prestige? A Public Choice Approach and the Case of Budapest", International Journal of Urban and Regional Research, 35 (4), p. 735 - 752.

Degórska B., 2012, „Spatial growth of urbanised land within the Warsaw metropolitan area in the first decade of the $21^{\text {st }}$ century", Geographia Polonica, Vol. 85 (3), p. 77 - 95.

Degórska B., DerĘgowska A., 2008, Zmiany Krajobrazu obszaru metropolitalnego Warszawy XX i XXI wieku, Atlas Warszawy, 10, IGiPZ PAN, Warszawa. (Traduction : changement du paysage métropolitain varsovien XX et XXI siècle).

Dumolard P., 2011, Données géographiques : analyse statistique multivariée, Lavoisier, Paris.

Enyedi G., 1998, „Transformation in Central European Postsocialist Cities », in Enyedi G. (dir.), Social Change and Urban Restructuring in Central Europe, Akadémiai Kiado, Budapest, p. 9 - 34.

Florida R., 2002, The Rise of the Creative Class, And How It's Transforming Work, Leisure, Community and Everyday Life, Basic Books, New York, $434 \mathrm{p}$.

Florida R., 2005, Cities and the Creative Class, Routledge, New York, London, 198 p.

GĄsior-Niemiec A., Glasze G., Lippok D., Pütz R., 2007, „Gradzenie Grodzenie miasta: casus Warszawy", Studia Regionalne i Lokalne, Nr 4(30), p. 7 -30, (Traduction: clôturer la ville : le cas de Varsovie).

Glock B., 2007, "Social and spatial consequences of the restitution of real estate", in K. Stanilov (dir.), The Post-Socialist City, Urban Form and Space Transformations in Central Europe after Socialism, The Geojournal Library, 92, Springer, the Netherlands, p. 191 - 214.

Górczyńska M., 2012, Changement dans la différentiation socio-spatiale des quartiers à Varsovie et dans l'agglomération parisienne : dynamiques et acteurs, Thèse de doctorat sous la direction de G.

WĘcławowicz et M. Berger, Institute of Geography and Spatial Organization Polish Academy of Sciences, Université Paris 1.

Górczyńska M., 2013, „Changes in the social composition in pre-war housing in Warsaw”, Intervention à l'AAG (Association of American Geographers) de Los Angeles le 11 avril 2013.

Hay I., 2013, Geographies of the super-rich, Cheltenham, Edward Elgar, London.

Higley J., Lengyel G. (dir.), 2000, Elites after State Socialism, Rowman \& Littlefield Publishers, inc., Lanham, Boulder, New York, Oxford.

Higley J., Pakulski J., 2000, “Elite theory and research in postcommunist societies”, in J. FrentzelZagórska, J.Wasilewski (dir.), The Second Generation of Democratic Elites in Central and Eastern Europe, Institute of Political Studies Polish Academy of Sciences, Warsaw, p. 37 - 49.

Higley J., Pakulski J., Wesołowski W. (dir.), 1998, Postcommunist Elites and Democracy in Eastern Europe, London: Macmillan.

Jałowiecki B., 2000, „Percepcja przestrzeni Warszawy”, Studia Regionalne i Lokalne, NR 2(2), p. 79 -100, (traduction: „Perception de l'espace varsovien”). 
Jałowiecki B., Krajewska M., Olejniczak K., 2004, „Klasa metropolitalna w przestrzeni Warszawy”, in J. Grzelak, T. Zarycki (dir.), Społeczna mapa Warszawy, Wydawnictwo naukowe Scholar, p. 132 146, (traduction: „La classe métropolitaine dans l'espace varsovien”, in Carte sociale de Varsovie).

Komornicki J., 2011, Premiany mobilność codziennej polaków na tle rozwoju motoryzacji, Prace Geograficzne nr 227, IgiPZ PAN, Warszawa, (traduction : Les changements des mobilités quotidiennes des Polonais par le développement de la motorisation).

Le Goix R., 2002, « Les gated communities à Los Angeles, place et enjeux d'un produit immobilier pas tout à fait comme les autres ", L'espace géographique, 2002, 4, 31, p. 328-344.

Le Goix R., 2006, « Les gated communities aux Etats-Unis et en France : une innovation dans le développement périurbain?", Hérodote, 3, n¹22, p. 107-137.

Liszewski S., 1999, „Elity intelektualne w przestrzeni miasta. Przykład Łodzi”, in J. Kaczmarek, Zróżnicowanie przestrzenne struktur społecznych $w$ dużych miastach, Konwersatorium Wiedzy o Mieście, XI, Katedra Geografii Miast i Turyzmu Uniwersytetu Łódzkiego. Łódzkie Towarzystwo Naukowe, Łódź, p. 9 - 21, (Les élites intellectuelles dans l'espace urbain. Exemple de Lodz, in Différenciation spatiale de la structure sociale dans les grandes villes).

Madoré F., 2009, « Le développement continu des complexes résidentiels clos », in T. Paquot (dir.), Ghettos de riches. Tour du monde des enclaves résidentielles sécurisées, Paris Perrin, p. 134-145.

Marcińczak S., Musterd S., StĘpniak M., 2012, "Where the Grass is Greener: Social Segregation in Three Major Polish Cities at the Beginning of the $21^{\text {st }}$ Century", European Urban and Regional Studies, 19, p. 383-403.

Marcińczak S., Tammaru T., Novák J., Gentile M., Kovács Z., Temelová J., Valatka V., Kährik A., Szabo B., 2015, "Patterns of Socioeconomic Segregation in the Capital Cities of Fast-Track Reforming Postsocialist Countries”, Annals of the Association Geographers, 105(1), p. 183 - 202.

Mosca G., 1953, (réed. 1884), Elementi di Scienzia Politica, Bari, Gius. Laterza.

Musil J., 2005, „Why socialist and post-socialist cities are important for forward looking urban studies, Presentation lors de la conférence "Forward Look on Urban Science", Helsinki, p. 26 - 28.

Pareto V., 1919, Traité de sociologie générale, éd. Française par P. Boven, Lausanne-Paris, Librairie Payot, 2 volumes.

Pfirsch T., «La localisation résidentielle des classes supérieures dans une ville d'Europe du Sud. Le cas de Naples », L’Espace géographique 4/2011 (Tome 40), p. 305-318

URL : www.cairn.info/revue-espace-geographique-2011-4-page-305.htm.

Pinçon M., Pinçon-Charlot M., 1989, Dans les beaux quartiers, Editions du Seuil.

Pinçon M., Pinçon-Charlot M., 1992, Quartiers bourgeois, quartiers d'affaires, Payot, « Documents », Paris.

Pinçon M., Pinçon-Charlot M., 1996, Grandes fortunes. Dynasties familiales et formes de richesse en France, Payot, « Documents », Paris.

Pinçon M., Pinçon-Charlot M., 2005a, Châteaux et Châtelains : les siècles passent, le symbole demeure, A. Carrière, Paris.

Pinçon M., Pinçon-Charlot M., 2005b, Voyage en grande bourgeoisie, Quadrige, Essais-Débats, Puf. Regulska J., 1987, “Urban development under socialism: the Polish experience”, Urban Geography, 8(4), p. 321-339. 
Śleszyński P., 2012, Warszawa i obszar metropolitalny Warszawy a rozwój Mazowsza. Trendy Rozwojowe Mazowsza, 8, Warszawa: Mazowieckie Biuro Planowania Regionalnego, (traduction : Varsovie, l'espace métropolitain de Varsovie et le développement de la Mazovie).

Słomczyński K. M., 2002, "Introduction: Social Structure, its Changes and Linkages", in Social Structure: Changes and Linkages: The Advanced Phase of the Post-Communist Transition in Poland, Warsaw: IFiS Publishers.

Tarrius A., 1992, Les fourmis d'Europe : Migrants riches, migrants pauvres et nouvelles villes internationales, L'Harmattan, Paris, 207 p.

Wagner A.-C., 1998, Les nouvelles élites de la mondialisation: Une immigration dorée en France, Presses Universitaires de France, Paris, 236 p.

WĘcławowicz G., 1996, Contemporary Poland, Space and Society, Changing Eastern Europe 4, UCL Press, London.

Zarycki T., 2008, Kapitał kulturowy, Inteligencja w Polsce i w Rosji, WUW, Warszawa, (Traduction: Le capital culturel, l'intelligentsia en Pologne et en Russie)

\section{APPENDIXES}

Annexe 1. Variables qualitatives utilisées pour l'analyse factorielle des correspondances

\begin{tabular}{|c|c|c|c|}
\hline Caractéristiques & Variables & Caractéristiques & Variables \\
\hline \multicolumn{2}{|c|}{ Catégorisation professionnelle } & \multicolumn{2}{|c|}{ 4. Pratiques } \\
\hline \multirow{3}{*}{ Type d'élite } & \multirow{3}{*}{$\begin{array}{c}\text { Cult_art } \\
\text { Economique } \\
\text { Cult_Rech } \\
\text { Politique }\end{array}$} & $\begin{array}{c}\text { Mode de déplacement } \\
\text { dans la ville }\end{array}$ & $\begin{array}{c}\text { Voiture } \\
\text { transp_commun }\end{array}$ \\
\hline & & \multicolumn{2}{|c|}{ 5. Définition et caractéristiques individuelles } \\
\hline & & \multirow{3}{*}{$\begin{array}{l}\text { Se définissant en tant } \\
\text { qu'élite }\end{array}$} & \multirow{3}{*}{$\begin{array}{c}\text { non_elit } \\
\text { oui_elit } \\
\text { pas_avis_elit }\end{array}$} \\
\hline Mode $d$ & propriété & & \\
\hline \multirow{2}{*}{$\begin{array}{c}\text { Disposant d'une } \\
\text { résidence secondaire }\end{array}$} & \multirow{2}{*}{$\begin{array}{l}\text { oui_ } 2^{\mathrm{e}} \text { _resid } \\
\text { non_2 } 2^{\mathrm{e}} \text { resid }\end{array}$} & & \\
\hline & & \multirow{2}{*}{$\begin{array}{l}\text { Se définissant en tant } \\
\text { qu'inteligent }\end{array}$} & \multirow{2}{*}{$\begin{array}{l}\text { oui_intel } \\
\text { non_intel }\end{array}$} \\
\hline \multirow{3}{*}{$\begin{array}{c}\text { Nombre de propriétés } \\
\text { dont il dispose en plus } \\
\text { de son logement de } \\
\text { résidence }\end{array}$} & \multirow{3}{*}{$\begin{array}{l}\text { propri_plus0 } \\
\text { propri_plus1 } \\
\text { propri_plus2 } \\
\text { propri_plus3 } \\
\text { propri_plus4_5 }\end{array}$} & & \\
\hline & & \begin{tabular}{|c|} 
L'entourage défini en tant \\
qu'élite
\end{tabular} & $\begin{array}{l}\text { elitentour_oui } \\
\text { elitentour_non }\end{array}$ \\
\hline & & \multirow{6}{*}{ Age de l'interviewé } & $\begin{array}{l}\text { 20_30ans } \\
31 \_40 \text { ans }\end{array}$ \\
\hline \multirow{4}{*}{$\begin{array}{l}\text { Age du bâti de la } \\
\text { résidence principale }\end{array}$} & avant_1945 & & 41_50ans \\
\hline & 1945_1970 & & 51_60ans \\
\hline & 1971_1988 & & 61_70ans \\
\hline & après_1989 & & 71_80ans \\
\hline \multirow{2}{*}{$\begin{array}{l}\text { Vivant dans une } \\
\text { résidence fermée }\end{array}$} & \multirow{2}{*}{$\begin{array}{l}\text { oui_ferm } \\
\text { non_ferm }\end{array}$} & & plus81ans \\
\hline & & \multirow{3}{*}{$\begin{array}{l}\text { Nombre de langues } \\
\text { étrangères connues }\end{array}$} & 1_2_lang \\
\hline Préf & nces & & 3_4_lang \\
\hline \multirow[b]{2}{*}{ Age du bâti } & \multirow{2}{*}{$\begin{array}{c}\text { pref_neuf } \\
\text { pref_ancien } \\
\text { pas_pref_na } \\
\end{array}$} & & 5_6_lang \\
\hline & & $\begin{array}{c}\text { Plus de } 6 \text { mois à } \\
\text { l'étranger }\end{array}$ & $\begin{array}{l}\text { 6mois_oui } \\
6 \text { mois_non }\end{array}$ \\
\hline Type de logement & $\begin{array}{l}\text { pref_maison } \\
\text { pref_appart } \\
\text { pas_pref_ma }\end{array}$ & \multirow[b]{2}{*}{ Revenu brut par mois } & $\begin{array}{c}\text { de3201z_a5600z } \\
\text { de5601z_a8000z } \\
\text { de8001z_a13000z }\end{array}$ \\
\hline Résidence fermée & \begin{tabular}{|c|} 
non_aim_ferm \\
oui_aim_ferm \\
pas_pref_aim_fer \\
m
\end{tabular} & & $\begin{array}{c}\text { de13001_a21800z } \\
\text { de21801z_a31500z } \\
\text { de31501z_a96600z } \\
\text { de } 96600 z\end{array}$ \\
\hline
\end{tabular}

Source : entretiens réalisés en 2011 


\section{NOTES}

1. Par exemple: les élites de la mondialisation d'A.-C.Wagner (1998), les classes créatives de R. Florida (2002, 2005) ou encore les néo-nomades de A. Tarrius (1992).

2. Par exemple : Higley et Pakulski, 2007 et Higley et alii, 1998.

3. A l'exception des travaux de T. Pfirsch (2011) sur les classes supérieures à Naples.

4. Ne sont prises en compte dans cette article que les résidences principales des interviewés, c'est-à-dire à Varsovie intra-muros.

5. A la fin de chaque entretien, il était demandé aux interviewés les coordonnées de cinq personnes qui répondaient à leur définition de l'élite et qui vivaient à Varsovie (aire métropolitaine). Seuls deux individus ainsi transmis vivaient dans la banlieue de Varsovie.

6. Certaines personnes interrogées m'ont été recommandées plusieurs fois durant mon investigation, alors même qu'il s'agissait de cercles professionnels différents.

7. En été $2013,1 €$ était égal à 4,22 PLN.

8. "Wodniesieniu do Europy Środkowej i Wschodniej, gdzie rola tradycyjnej kultury arystokratycznej w życiu społecznym jest obecnie znikoma, mówić można bardziej o postarystokratycznym, a wiĘc odziedziczonym po arystokracji, wymiarze kapitału kulturowego ucieleśnionego wymieszanym z wartościami kultury wysokiej współczesnych krajów zachodnich."

9. BDL (Bank Danych Lokalnych), GUS, 2002.

10. Selon Glasze (2001: Górczyńska, 2012), il existerait deux types de résidences fermées : les condominiums (résidences luxueuses du centre-ville avec digicode) et les résidences fermées (gated-estates) avec clôture composées soit de maisons, de maisons jumelées ou d'immeubles.

11. L'interprétation s'effectue selon la proximité et l'éloignement d'un point-ligne i par rapport à un point-colonne $\mathrm{j}$, mais aussi par rapport à l'éloignement d'un point par rapport à l'origine (écart à l'indépendance) (Dumolard, 2011). Ainsi, lorsque deux variables sont proches, c'est qu'elles sont sur-représentées pour caractériser un individu particulier. Par ailleurs, si deux variables sont très éloignées l'une de l'autre, un individu présentant une caractéristique détient très rarement l'autre.

12. Ce pourcentage de confiance est tout à fait acceptable pour une analyse s'appuyant sur des données qualitatives à l'échelle des individus.

13. A partir de la moitié des années 1960 , afin de parer à la pénurie de logements en Pologne, les coopératives d'habitation ont pris le relais de la construction de logements communaux sous étroit contrôle de l'Etat, devenant ainsi les principaux promoteurs immobiliers dans les espaces urbains jusqu'au début des années 1990 (Bouloc et Górczyńska, 2014).

14. Sur ce blog internet http://jacek23151.pinger.pl/m/10879729 (consulté le 14/03/2015), des photos de la rue Ludwika Narbutta (vieux Mokotów) sont présentées et permettent de rendre compte de l'architecture ancienne présente dans ce quartier. Un panorama plus complet de la diversité du bâti de Mokotów est visible sur ce forum http://www.skyscrapercity.com/ showthread.php?t=1655562\&page=10 (consulté le 14/03/2014).

15. L'origine du graphique représente les caractéristiques qui sont partagées par la majorité des individus interrogés. 


\section{ABSTRACTS}

After 1989, the real estate offer in Poland became greatly diversified. Elites, considered as main winners of the politico-economic transformation, commenced to seek for a dwelling corresponding to their social status and allowing them to stand out from other social groups. In Warsaw, despite some resemblances in their residential trajectories, preferences and choices, the different profiles within the group of elites may be distinguished. This article attempts to go beyond the classical differentiation of elites by their occupation and proposes an original typology of elites regarding socio-economic, demographic and residential characteristics, life styles as well as residential preferences. The diversity of the elitist housing has become one of the components of the process of distinction between different fractions in the group of elites.

Après 1989, l'offre immobilière s'est extrêmement diversifiée en Pologne. Les élites, grandes gagnantes de la transformation politico-économique, cherchent à emménager dans des logements correspondant à leur statut social et permettant de les distinguer du reste de la population. Malgré quelques similitudes dans leurs trajectoires, préférences et choix résidentiels, des profils types se dessinent au sein des individus considérés comme des élites à Varsovie. Cet article essaie de dépasser la différenciation classique des élites selon les professions proposant une typologie originale de celles-ci selon des caractéristiques socio-économiques, démographiques, d'habitation, de choix de vie et de préférences résidentielles. La diversité de l'habitat élitaire devient alors une composante de cette distinction existante entre les différentes élites.

Nach 1989 hat sich der Immobiliensektor grundlegend verändert. Die Eliten des Landes, die am meisten vom politischen Umbruch profitierten, wollten sich in ihrem Wohnraum vom Rest der Gesellschaft stärker differenzieren. In Warschau lassen sich verschiedene Strömungen innerhalb der Eliten unterscheiden. Unterschiedliche Präferenzen in der Immobilienauswahl haben das Stadtbild stark beeinflusst. Dieser Artikel löst sich von der klassischen Analyse der Elitenbildung und bietet eine eigenständig entworfene Typologie, die sich auf sozioökonomische und demographische Kriterien, geschmackliche Präferenzen und Lifestyle-Gewohnheiten der oberen Gesellschaftsschicht bezieht. Wohnen ist aber auch innerhalb der Eliten zu einem grundlegenden Unterscheidungsmerkmal geworden.

\section{INDEX}

Mots-clés: comportements résidentiels, habitat élitaire, Pologne, trajectoires résidentielles, typologie des élites, Varsovie

Schlüsselwörter: Eliten-Typologie, Polen, Warschau, Wohn Bahn, Wohn Verhalten, Wohnraumgestaltung der Eliten

Keywords: elites' housing, elites' typology, Poland, residential behaviours, residential trajectories, Warsaw

\section{AUTHOR}

\section{CAROLINE BOULOC}

Univ Lyon, CNRS, ENS de Lyon, EVS, UMR5600, F-69007, France, caroline.bouloc1@ens-lyon.fr 